\title{
Germanica
}

\section{Heinrich Heine, le génie de l'amour-haine}

\section{Marcel Reich Ranicki}

Traducteur : Bénédicte Terrisse

\section{(2) OpenEdition \\ Journals}

Édition électronique

URL : https://journals.openedition.org/germanica/8157

DOI : 10.4000/germanica.8157

ISSN : 2107-0784

\section{Éditeur}

Université de Lille

\section{Édition imprimée}

Date de publication : 1 décembre 2019

Pagination : 127-138

ISBN : 978-2-913857-44-5

ISSN : 0984-2632

Référence électronique

Marcel Reich Ranicki, « Heinrich Heine, le génie de l'amour-haine », Germanica [En ligne], 65 | 2019, mis en ligne le 01 janvier 2022, consulté le 08 janvier 2022. URL : http://journals.openedition.org/ germanica/8157; DOI : https://doi.org/10.4000/germanica.8157 


\section{Heinrich Heine, le génie de l'amour-haine}

Marcel Reich-Ranicki ${ }^{1}$

1.

Écrire sur Heine en Allemagne reste encore une chose délicate et périlleuse. Celui qui dit qu'on le surestime, qui le remet en question ou même le rejette, ne tient pas pour superflu, aujourd'hui encore, de prendre ses distances, ne serait-ce qu'en passant, avec ceux qui combattirent surtout le Juif Heine. Inversement, celui qui évoque sa grandeur et son caractère exceptionnel doit toujours craindre qu'on le confonde avec ceux qui, pour quelque raison que ce soit, mènent une politique de réparation par des moyens littéraires.

Ainsi, aujourd'hui encore, semble-t-il, la fumée des autodafés et des chambres à gaz continue d'obscurcir notre vue. En tout cas, les premiers auspices sous lesquels on recommença à s'intéresser à Heine après 1945 étaient loin de la sobriété critique et de l'objectivité scientifique. Car, bien sûr, pas plus que la haine des Juifs, la réparation n'est une catégorie adaptée à l'élucidation d'un phénomène littéraire.

En revanche, il est frappant de constater qu'il y a cinquante ou cent ans déjà, les discussions autour de Heine ne se caractérisaient en général pas par la sobriété ni l'objectivité, mais par des émotions exacerbées et un ressentiment profond. Depuis toujours en Allemagne, Heine

1. - Nous remercions ici Thomas Anz de nous avoir accordé les droits de traduction pour ce texte.

GERMANICA, 2019, LXV, pp. 127 à 144. 
déclenche des propos enflammés : même avant Auschwitz, son cas se trouvait toujours sur le fil du rasoir. Mais ce qui autrefois compliquait considérablement la discussion sur Heine et la complique encore actuellement, est, comme tout ce qui le concerne, inséparable de sa judéité, tout en étant à chercher à un autre niveau - par-delà toute forme de diabolisation antisémite et toute glorification philosémite.

\section{2.}

Aucun écrivain allemand n'a suscité de réactions aussi violentes de son vivant que Heine. À l'exception de Goethe, aucun poète allemand, de loin, n'a bénéficié d'une popularité aussi grande. De même, l'histoire de la littérature allemande ne connaît pas de semblable exemple d'une réception posthume si agitée, ni si passionnée, ni bien sûr si ambivalente : aucun des grands poètes allemands n'a été plus copieusement insulté, n'a été combattu avec plus d'obstination. Aucun n'a donné plus souvent prise à des polémiques si acharnées, où il en allait de questions aussi décisives pour le cours du monde que de savoir s'il fallait donner son nom à une rue ou à une université ou s'il fallait l'honorer par un monument ou une simple plaque commémorative. Aucun poète allemand n'a trouvé pareil écho à l'étranger, aucun n'a été si souvent ni si radicalement attaqué ou défendu au moyen d'arguments démagogiques et de citations tronquées.

Mais rien ne serait plus inconsidéré que de prétendre que tout cela témoigne de sa grandeur. Car la popularité de Heine ne prouve en aucun cas la qualité de ses vers : il est arrivé que ce soient précisément les plus faibles, les vers complaisants et routiniers, qui soient les plus aimés et les plus imités. Quant au succès de sa poésie à l'étranger, il le doit en grande partie au fait qu'elle est facilement traduisible en langues étrangères. En faire le reproche à Heine - et c'est ce qu'on a souvent fait - est tout bonnement insensé. D'un autre côté, cependant, la traductibilité d'un poème peut difficilement être un critère de qualité. L'échelle de sa poésie - cela me semble absolument certain - va du poème génial au pur produit d'artisanat. En outre, il ne faut pas perdre de vue que si la violence et la démagogie qui caractérisent la lutte autour de Heine ne peuvent certainement pas être justifiées par ses défauts manifestes, ils n'y sont toutefois pas étrangers.

Ce fut un provocateur né et un éternel fauteur de troubles. Il mit le doigt sur les blessures les plus douloureuses de ses contemporains sans réfléchir aux conséquences qui allaient nécessairement en découler pour lui. Cela le préoccupait très peu de savoir qu'il offrait aux autres une cible très facile, et ceci pas seulement parce qu'il adorait les jugements extrêmes et donc souvent contestables. Il n'assurait jamais ses arrières, les mesures de précaution étaient peu compatibles avec son 
tempérament. De fait, il se battait à visage découvert. On pourrait même dire : il partit en exil pour ne jamais avoir à se mettre à couvert.

Il fut un virtuose de la polémique. Mais de tact et de tactique, il ne voulut rien savoir. Il semblerait presque qu'il fût incapable de séparer le sujet traité de la personne impliquée ; en tout cas, cela ne lui a jamais importé. Il aurait pu se permettre de renoncer aux plaisanteries puériles et aux mots d'esprit méchants, aux arguments faciles et aux piques malveillantes. Mais il ne cessait d'y avoir recours, même là où on ne l'avait pas provoqué. Rien ne le retenait d'accuser ses ennemis (et pas seulement eux) d'impuissance et d'homosexualité, et d'énumérer toutes les sortes d'infirmités physiques (allant jusqu'à la diarrhée) dont ils souffraient selon lui. Aussi injustement qu'on l'eût traité - de son vivant mais aussi plus tard -, lui-même ne fut pas moins injuste. C'est ainsi qu'il pourvut d'un matériau riche et souvent efficace presque automatiquement tous ceux qu'il avait attaqués et tous ceux qui, pour d'autres raisons, voulaient écrire contre lui. Cet admirable buteur réalisa nombre de buts contre son propre camp et s'en accommoda placidement.

À cela s'ajoute le goût louable de Heine pour la concision aphoristique, les formulations spirituelles et acérées, consciemment outrées et, par-là, particulièrement marquantes. Elles font de ses vers et de sa prose une matière que l'on peut extraordinairement bien citer. Et avec des citations de Heine, on peut sans peine prouver beaucoup de choses. Seulement, en règle générale, justement parce qu'il est si facile de se servir de ses déclarations, on n'y gagne pas grand-chose.

Car si les réactions contradictoires et souvent agressives par rapport à Heine ont été favorisées et intensifiées par des circonstances nombreuses et très diverses, elles tirent leur origine profonde de son caractère particulier qui, cependant - et c'est surtout cela qui est important - ne se manifeste pleinement dans aucun de ses écrits. Il n'y a aucun livre de Heine qui serait aussi représentatif que, par exemple Faust, ou La Montagne magique, ou Le Procès pour leurs auteurs. Si l'on veut lui rendre justice, il faut absolument voir son œuvre trouble et inégale, ambivalente et incomparable - semblable en cela du reste à celle de Brecht comme un tout. Elle consiste en de nombreuses, pour la plupart petites, parties et se révèle finalement être une unité.

Parfaite, l'œuvre de Heine ne l'est certainement pas. Mais elle représentait à son époque une chose inouïe, parfaitement scandaleuse. Ses écrits forment les fragments d'une seule et unique provocation.

3.

La biographie de Heine va du moyen-âge juif à la modernité européenne. L'œuvre de Heine nous conduit du romantisme allemand à la modernité der Allemands. 
Lui seul réussit ce qui après l'ère Goethe et Schiller, Kleist et Hölderlin était absolument nécessaire : la dépathétisation radicale de la poésie allemande. Il la libéra du sublime et du digne, de l'hymnique et du solennel et aussi de l'obscur. Et il lui donna ce dont elle avait privé le plus le lecteur allemand : la légèreté et la grâce, le charme et l'élégance, l'humour et l'esprit, la rationalité et l'urbanité et, à l'occasion, aussi la frivolité.

Que le chant et la pensée ne s'excluent pas forcément, on le savait déjà avant Heine, et d'autres avaient déjà démontré que, même dans les contrées germaniques, il est possible d'être un poète et en même temps un penseur. Mais Heine fut le premier à réaliser la synthèse impeccable de poésie et d'intellect sans surcharger la poésie de philosophie - comme c'était le cas la plupart du temps en Allemagne. Il a renouvelé et enrichi le vers allemand par le langage courant, le vocabulaire du quotidien, sans lui ôter pour autant son caractère poétique. Et il a vivifié et intensifié la prose allemande avec des sonorités, des images et des rythmes lyriques, sans pour autant la poétiser.

Lorsque, dans son pamphlet tristement célèbre, Karl Kraus écrivait de « ce Heinrich Heine », qu'il avait « tellement desserré le corsage de la langue allemande qu'aujourd'hui tous les commis [...] peuvent tripoter ses seins $\gg^{2}$, il attirait l'attention, certes sans le vouloir, sur l'une des grandes réalisations de Heine. Car la langue allemande à l'époque se languissait de celui qui se dévouerait enfin pour lui desserrer le corsage.

" Le caractère courant et l'évidence qu'il emprunte à la langue de la communication » étaient - pensait Adorno - «le contraire de la sécurité procurée à celui qui habite dans la langue ». C'est possible, mais c'est parce que « la sécurité procurée à celui qui habite dans la langue » lui a été refusée, qu'il a pu acquérir cette distance à la langue lui permettant de la traiter comme un instrument. Ce qu'Adorno tenait pour « l'absence de résistance [de Heine] contre le mot courant $»^{3}$ était, en réalité, cette extraordinaire sensibilité à la douleur à laquelle nous devons un renouveau radical aussi bien de la langue de la poésie que de la prose.

2. - Traduction légèrement modifiée par nous : Karl Kraus, « Heine et les conséquences », trad. Éliane Kaufholz, in : Éliane Kaufholz (dir.), Karl Kraus, Paris, Éditions de l'Herne, 1975, p. 52-64, p. 56. Texte original en allemand : Karl Kraus, « Heine und die Folgen », Die Fackel n`329/30, 1910. Référence indiquée par Marcel Reich-Ranicki : Karl Kraus, Auswahl aus dem Werk, Auswahl von Heinrich Fischer, München, KöselVerlag, 1957, p. 187.

3. - Theodor W. Adorno, « La blessure Heine », in : Theodor W. Adorno, Mots de l'étranger et autres essais. Notes sur la littérature II, trad. Lambert Barthélémy et Gilles Moutot, Paris, Éditions de la Maison des sciences de l'homme, 2004, p. 3641, p. 39. Texte original en allemand indiqué par Marcel Reich Ranicki : Theodor W. Adorno, « Die Wunde Heine » (1956), in : Noten zur Literatur, Frankfurt a. M., Suhrkamp Verlag, 1958, p. 148-149. 
En modernisant la langue de la littérature allemande et en lui ôtant ses oripeaux, Heine créa la condition préalable la plus importante à la démocratisation qu'il fut lui-même - comme aucun autre écrivain du dix-neuvième siècle - en mesure de réaliser. Ce dont les meilleurs de ses prédécesseurs avaient rêvé, il le réussit : surmonter le fossé entre l'art et la réalité, entre la poésie et la vie.

C'est dans ce contexte que s'inscrivent aussi les mérites de Heine en matière de journalisme. Il est vrai que, précisément sur ce terrain, le nombre de ses péchés semble particulièrement grand et qu'il a répandu plus d'une habitude fâcheuse dont souffre la presse allemande encore aujourd'hui. Mais il est celui qui a montré qu'un seul et même homme pouvait être, dans le même temps, un poète génial et un journaliste de presse écrite professionnel.

Lui, le journaliste le plus important parmi les poètes allemands et le poète le plus célèbre parmi les journalistes du monde entier, fut, du moins en Allemagne, le premier à avoir reconnu les possibilités offertes par la presse moderne et à avoir su constamment en faire usage. C'est précisément cela qui lui a valu le plus d'ennemis. On craignait ses pensées et ses opinions, c'est certain, mais on craignait encore plus sa capacité à exprimer ces pensées et opinions d'une manière telle qu'elles devinssent plausibles et intéressantes pour d'innombrables lecteurs.

Le journalisme d'aujourd'hui continue d'utiliser nombre des moyens et formes éprouvés par lui et vit pour une grande part de ses trouvailles. Et de même qu'on ne peut plus penser le drame allemand moderne sans ce que Büchner a accompli, de même il est difficile de se représenter la poésie allemande du vingtième siècle - de Brecht et Benn à Grass et Enzensberger - sans l'influence de Heine. Que les écrivains qui lui doivent beaucoup parmi ceux qui sont venus après lui n'en aient souvent pas été et n'en soient toujours pas conscients, ne change rien à la chose.

\section{4.}

Depuis des années et des décennies, communistes et anticommunistes se réclament de Heine. Dans ce cadre, on se réfère en général à ses écrits tardifs, notamment à ses Aveux de 1854 et à l'avant-propos au Lutetia de 1855. Dans ces deux écrits on trouve des propos hautement dignes d'être suivis, si ce n'est que chaque parti aime choisir ce qui l'arrange, et lorsqu'il arrive que les deux partis citent les mêmes passages, c'est pour y souligner des membres de phrases différents.

C'est ainsi que l'on se réfère fréquemment à ce constat remarquable des Aveux, selon lequel « les chefs plus ou moins occultes des communistes allemands » étaient « les têtes les plus capables, les caractères les plus énergiques de l'Allemagne ». Ces « docteurs de la révolution», 
Heine les tenait pour « les seuls hommes en Allemagne qui aient vie $»^{4}$, c'est à eux, croyait-il, qu'appartenait l'avenir. Cependant, il inséra dans cette prédiction les mots « je le crains ».

L'ensemble est un jeu quelque peu vain pour la simple raison que Heine, comme tous ceux qui, au milieu du siècle passé, eurent recours au concept de " communisme », voulait dire par-là et ne pouvait que vouloir dire quelque chose d'autre que nous aujourd'hui. On ne peut attendre aucune prise de position de Heine par rapport au marxisme parce que le marxisme n'existait pas encore. De même, renvoyer de manière polémique à ses opinions positives ou négatives sur le communisme fait peu de sens, parce qu'à l'époque, c'est-à-dire peu de temps après la publication du Manifeste communiste, ce dernier était à peine plus qu'une idée esquissée à grands traits, une vision aussi noble que vaine. De réalité politique, on ne pouvait encore parler.

D'une certaine manière pourtant, ce jeu est révélateur : il montre que Heine ne se laisse pas confondre avec un programme, une idéologie ou une organisation. Car ce qui ressort de ce conflit entre communistes et anticommunistes vaut pour tous les mouvements politiques et idées auxquels Heine s'est intéressé. Il réussit à résister à toute fixation qui aurait limité sa liberté de mouvement et à rester un solitaire.

Il prouva - et à l'époque, c'était quelque chose de neuf et d'étonnant - que, depuis sa position d'écrivain indépendant, il était possible de combattre efficacement ce qu'aujourd'hui nous appelons l'establishment. En d'autres termes : qu'il était possible d'être un écrivain politique sans devenir un homme politique écrivant de la poésie. C'était un homme de lettres engagé mais qui ne voulait rien savoir de la littérature à thèse. Il se moquait d'elle parce qu'il la tenait pour inutile. Même là où une influence politique directe et rapide lui importait, il ne consentait pas à des concessions artistiques. Son œuvre contredit l'affirmation selon laquelle il est nécessaire de se retirer dans sa tour d'ivoire pour rester un véritable artiste.

Tout compte fait, il était l'écrivain de gauche typique. Cependant il exécrait le radicalisme de gauche et le méprisait profondément. Et il le craignait. Tôt, les pensées matérialistes le fascinèrent. «Avez-vous compris l'idée d'un repas de midi, mon cher ? Celui qui a compris cette idée, comprend toute l'activité humaine $»^{5}-$ c'est ce que l'on peut déjà

4. - Henri Heine, «Les Aveux d'un poète », Revue des Deux Mondes, t. 7, 1854, p. 1182. Référence du texte original en allemand donnée par Marcel Reich-Ranicki : Heinrich Heine, Sämtliche Werke, Hans Kaufmann (dir.), vol. XIII, München, Kindler Verlag, 1964, p. 245-246.

5. - Nous traduisons. Référence du texte original donné par M. Reich-Ranicki : Heinrich Heine, ibid., vol. VI, p. 147. 
lire dans ses Lettres de Berlin publiées en 1822. Heine semble n'avoir jamais oublié cela.

Dans la tragédie William Ratcliff imprimée l'année suivante, le jeune Heine laisse son héros éponyme parler de ceux qui nagent dans la surabondance :

Et regarder avec mépris le meurt-de-faim,

Qui, sa dernière chemise sous le bras,

S'en va, trainant le pied et poussant des soupirs, frapper à la porte du mont-de-piété.

Oh ! voyez-les donc ces gens sages, et, bien repus,

Comme ils s'entourent d'un rempart de lois,

Pour éloigner les importuns,

Les affamés qui jettent des cris de détresse sous le fardeau de leur misérable vie !

Malheur à qui franchit ce rempart !

Tout est prêt, juges, bourreaux et la corde et la potence -

Et bien, il y a parfois des gens qui n'ont pas peur de tout cela.

Ce à quoi on lui répondit :

C'était aussi ma manière de voir, et je partageais les hommes

En deux nations qui se font une guerre sauvage,

D'un côté les repus, de l'autre les affamés ${ }^{6}$.

De même que Heine se battit toute sa vie pour des réformes sociales, il ne se lassa pas de défendre le plaisir contre la morale de la société et l'hypocrisie, et de réclamer qu'on libère l'éros d'une contrainte contre nature. C'est précisément à notre époque, alors que l'émancipation érotique commencée le siècle passé semble achevée, qu'il ne faut pas oublier que Heine fait partie de ceux qui ont initié ce processus contre la très forte résistance de l'opinion publique, notamment des Églises chrétiennes, et qui l'ont soutenu de manière efficace.

Mais quoi qu'il revendiquât et combattît, on ne pouvait jamais l'accuser de dogmatisme, jamais il n'était intolérant ou fanatique. C'est peutêtre à mettre en rapport avec le fait qu'il partageait pour l'essentiel les objectifs de Marx et Engels tout en rejetant leurs moyens d'y parvenir. Il était - malgré la diversité de ses déclarations sur cette question - sans aucun doute un adversaire de la révolution. Son véritable élément était l'ambivalence, celle qui, bien sûr, n'a rien à voir avec la réconciliation, ni même l'hésitation. C'était une ambivalence militante, agressive. Il

6. - Henri Heine, «William Ratcliff », in : Euvres Complètes. 5. Drames et fantaisies, Paris, Michel Lévy frères / librairie italienne, 1865, p. 180. Nous avons rétabli le retour à la ligne présent dans la version originale allemande, absente dans la traduction de Heine. Référence au texte original donnée par M. Reich-Ranicki : Heinrich Heine, Sämtliche Werke, op. cit., vol. IV, p. 234. 
était un génie de l'amour-haine - et il ne haïssait et n'aimait personne tant que les Allemands et les Juifs.

Heine éprouvait une jouissance pour ainsi dire voluptueuse à dire leur vérité à tous : aux Juifs et aux antisémites, aux Allemands et aux germanophobes, aux nobles et aux bourgeois, aux catholiques et aux protestants, aux poursuivants et aux persécutés, aux poètes du romantisme tardif et aux représentants de la Jeune Allemagne. Il s'asseyait constamment entre deux chaises. Et il me semble presque que c'est toujours là qu'est sa place.

Peut-on s'étonner qu'il soit entouré d'ennemis ? Il les rendait littéralement fous parce qu'il ne cessait de leur faire la démonstration de ce à quoi ils ne parvenaient la plupart du temps pas à s'élever : l'indépendance. Qu'il ait reçu de l'un ou de l'autre côté des subsides est chose certaine et on le lui a souvent reproché. Mais personne n'a pu prouver qu'il ait jamais fait la moindre concession en échange. Non, Heine n'était pas au service d'un prince, d'un gouvernement ou d'une autorité, il ne faisait allégeance à aucun parti, aucune Église ni aucun journal, il n'avait ni seigneur ni commanditaire. Bien qu'il soit un auteur politique et porte un regard critique sur son époque, il était uniquement responsable devant lui-même.

Bien sûr, il y avait déjà eu avant lui des écrivains indépendants. Heine fut cependant le premier à comprendre l'existence de l'écrivain indépendant comme une fonction et une institution. Et à faire considérer et respecter cette institution dans l'opinion publique allemande. Mais c'était là une scandaleuse provocation, d'autant plus s'agissant d'un Juif qui, de surcroît, exercera son activité pendant de nombreuses années depuis l'étranger. À l'époque, c'était une provocation. À l'époque seulement?

\section{5.}

Quiconque écrit sur Heine en croyant pouvoir faire abstraction du fait qu'il était juif - ou bien minimise ce fait - passera, j'en suis convaincu, à côté de son sujet. Ce n'est pas seulement sur le parcours de Heine, mais c'est sur toute son œuvre que son origine eut une influence directe ou indirecte, en tout cas extraordinairement forte. Il ne pouvait en être autrement. Il est possible que ce constat soit très banal, cependant cet aspect reste encore sous-estimé.

Il ne faut pas oublier que ce n'est que du vivant de Heine que commença l'émancipation des Juifs allemands et que, pour de nombreuses raisons, elle s'accomplit d'abord de manière lente et hésitante. Il fit ainsi partie de la première génération réchappée du ghetto. En ce sens, il était un nouveau venu et un parvenu - et fut traité comme tel par son entourage, qui, bien sûr, était majoritairement allemand. 
Il voulait être allemand. Mais il semble avoir compris très tôt qu'on ne l'y autoriserait pas. L'étudiant d'à peine vingt-deux ans qu'était Heine désigna ainsi - dans le petit essai « Le romantisme » - la langue allemande comme étant « notre bien le plus sacré », car elle est « une patrie même pour celui auquel la sottise et la perfidie la refusent $\rangle^{7}$.

Dans un tout autre contexte, il écrivit de nombreuses années plus tard que les Juifs « avaient transporté [la Bible] avec eux dans toutes les pérégrinations de l'exil pour ainsi dire comme une patrie portative $»^{8}$. - C'est certain, l'allemand - la langue, la littérature, la philosophie, l'histoire - s'avéra, comme Heine le souligna à plusieurs reprises, vital. Il fut cependant obligé de se créer à partir de l'allemand ni plus ni moins qu'une « patrie portative ». Cette situation particulière du Juif à l'intérieur de la société allemande, Heine fut capable de la sublimer et de la compenser et - comme aucun autre avant lui et comme un seul autre après lui : Kafka - de l'utiliser pour son œuvre. Il fit ainsi de nécessité vertu, la question de savoir à partir de quel moment et dans quelle mesure il s'agit d'un processus conscient étant ici sans importance.

En 1824, le jeune Heine fit la remarque que son deuxième livre les tragédies Almansor et Ratcliff avec un intermède lyrique - était, à la différence de son premier livre, « seulement intérieurement allemand ». Et d'expliquer : " Il est probable qu'en raison de sa mauvaise humeur contre l'allemand, ma muse ait coupé sa robe de manière un peu exotique $»^{9}$. Ce qu'il appelait mauvaise humeur n'était rien d'autre que la distance critique qui lui était imposée par son entourage allemand. Mais cette dernière l'aida à surmonter ce dont souffrait une grande partie de la littérature allemande de son époque : le provincialisme.

Rien d'allemand n'était étranger au Juif Heine, l'Allemagne cependant le força à devenir davantage qu'un auteur allemand. Ce qu'un Mörike, un Eichendorff ne parvinrent pas à faire, il est vrai qu'ils n'en avaient pas non plus un besoin absolu, il le réussit : il devint un écrivain européen - à peu près le seul que l'Allemagne eut entre Goethe et Thomas Mann. À quel point il souffrit de sa situation, de sa judéité et de sa germanité, on ne peut que le deviner. On le tint pour un poseur et un comédien. Effectivement, il lui arriva de trouver refuge dans le cabotinage. Et il avait besoin de poser pour la galerie pour endurer la vie. Il stylisa sa douleur pour la supporter. Par moments, la stylisation devenait gênante. Mais la douleur n'en était pas moins authentique.

7. - Nous traduisons. Référence au texte original donnée par M. Reich-Ranicki : Heinrich Heine, Sämtliche Werke, op. cit., vol. VII, p. 163.

8. - Henri Heine, «Les Aveux d'un poète », op. cit., p. 1191. Référence au texte original donnée par M. Reich-Ranicki : Heinrich Heine, Sämtliche Werke, op. cit., vol. XIII, p. 128.

9. - Nous traduisons. Référence au texte original donnée par M. Reich-Ranicki : Heinrich Heine, Briefe, Mainz, Florian Kupferberg Verlag, 1965, p. 150. 
Le plus souvent, il chercha un abri dans l'ironie. Même ce que d'aucuns prirent pour du cynisme n'était qu'un masque ou un refuge. Il n'était ni un cynique ni un dandy coquet ou un troubadour vaniteux - même si, à l'occasion, il se complaisait dans ces rôles. En réalité il fait partie - à l'instar de Kleist et de Hölderlin, de Kafka - de ces figures solitaires et déchirées, de ces figures tragiques de la littérature allemande.

Nulle part la solitude du Juif Heine parmi les Allemands n'apparaît plus fortement, nulle part son désespoir ne transparaît plus clairement que précisément dans cette partie de son œuvre où il n'est absolument pas question de Juifs - dans sa poésie érotique. Elle parle sans cesse contrairement à la poésie de Goethe - de dépit amoureux et d'amour malheureux, des souffrances de celui qui est dédaigné et éconduit. Se distinguent-elles donc des tourments d'un amoureux non juif? Non, évidemment pas. Le seul fait que des générations entières d'Allemands, de Français et de Russes, qui, on le sait, dans leur très grande majorité n'étaient pas des Juifs, aient pu reconnaître dans son Livre des Chants leurs expériences les plus intimes et que pendant un siècle, les amoureux se soient laissés conduire, ou si vous préférez, séduire par les vers de Heine, prouve qu'il avait trouvé comment exprimer les sentiments de millions de personnes. Mais cela n'a rien à voir avec sa judéité.

La situation particulière du Juif isolé dans la société, se battant pour la reconnaissance de sa citoyenneté, du nouveau venu ou du parvenu accablé de complexes et craignant sans cesse la résistance ou les chicanes de son environnement, accrut et décupla encore ses souffrances amoureuses. Cette situation spécifique, pour laquelle Heine possédait la sensibilité la plus fine et la plus à vif, donna à sa poésie érotique un accent nouveau et incisif, dimension inconnue jusqu'alors.

Pendant que les autres - Max Brod l'a fait remarquer - purent échapper à de telles souffrances en se réfugiant malgré tout dans une communauté, cette issue était interdite au Juif qui voulait être allemand. Car « Heine est seul, sans appartenance. Le judaïsme, au stade d'évolution qui est le sien à l'époque, n'offrait aucune possibilité de s'intégrer, aucun réconfort. La situation juive aggrava encore plus l'affect de la solitude que l'amour méprisé suscitait $»^{10}$.

L'aliénation du Juif apparaît ainsi comme le fond caché de la poésie érotique de Heine : l'amour désespéré devient le symbole de la situation de celui qui est exclu, repoussé et apatride. Comme dernière arme de défense et bouée de sauvetage, il lui restait ici encore l'ironie, ou plus exactement, l'autodérision mélancolique. Il en résulta ce qui avait rendu Heine célèbre dans le monde entier : le ton élégiaque et rebelle des vers

10. - Nous traduisons. Référence au texte original en allemand donnée par M. ReichRanicki : Max Brod, Heinrich Heine, Amsterdam, Verlag Allert de Lange, 1955, p. 269. 
qui exprimaient son désespoir avec la plus grande force de suggestion, mais sans hystérie, tout en le relativisant avec esprit et élégance.

À la judéité de Heine est liée aussi l'histoire contradictoire et hautement ambivalente de la réception de son œuvre en Allemagne. Ne nous faisons pas d'illusion : son œuvre n'a été que partiellement reçue, et de manière très limitée, pour finir par n'être justement pas intégrée. Parce que Heine était juif? La judéité du compositeur Mendelssohn n'a jusqu'en 1933 pas fait obstacle à la popularité de son concert pour violons ni à sa musique pour le Songe d'une nuit d'été. Berthold Auerbach fut, bien que Juif, l'un des plus grands écrivains à succès de son temps. Finalement, beaucoup de vers de Heine, notamment ceux que Schubert et Schumann, Mendelssohn-Bartholdy, Brahms et Hugo Wolf avaient mis en musique, furent acceptés avec enthousiasme par le public allemand.

Mais, autant il est certain que, par quelques-uns de ses écrits, Heine correspondait aux attentes des lecteurs et répondait, d'une manière parfois extrêmement douteuse, à leur goût, autant il est sûr que pour la partie la plus grande et de loin la plus importante de son œuvre - sa poésie tardive et toute sa prose - il suivit une voie propre et originale. Il ne prêta pas attention aux habitudes de réception du public allemand. Ce qu'il lui imposait, le public le ressentit manifestement comme une chose étrangère et choquante. Il refusait qu'on le brusque.

Ce n'est donc pas le Juif qu'on rejetait, mais le Juif provocateur, l'éternel fauteur de troubles. Il faudrait cependant être aveugle pour ne pas voir que l'originalité et la particularité de l'œuvre de Heine allaient de pair avec son origine et sa situation en tant que représentant de la première génération de Juifs allemands émancipés.

6.

En Allemagne, écrire sur Heine, c'est toujours écrire pour ou contre Heine. On ne l'a pas encore remisé au musée, le débat n'est pas encore terminé. Ainsi, Heine - comme Karl Marx, comme Richard Wagner continue d'exercer une influence jusque tard dans le vingtième siècle.

Il me semble à propos, en effet, de comparer l'importance qu'exerce pour la littérature le poète et auteur satirique, l'auteur politique et le journaliste qu'a été Heine avec celle que continuent d'avoir Marx pour la philosophie allemande et Wagner pour la musique allemande. À cette différence près que le génie de Heine n'a pas encore été tout à fait reconnu.

Mais que son œuvre continue d'inquiéter, qu'elle soit encore ce qu'elle était, une provocation et un scandale, n'est pas la moindre de ses qualités.

1972

Traduit de l'allemand et annoté par Bénédicte Terrisse Université de Nantes, CRINI 



\title{
« Heinrich Heine, le génie de l'amour-haine », une critique révélatrice. Réflexions autour d'une constellation textuelle et d'un mode de lecture
}

\author{
Bénédicte Terrisse \\ Université de Nantes
}

À de nombreux égards, le texte que nous avons choisi de traduire est représentatif du critique littéraire Marcel Reich-Ranicki. Au niveau le plus formel, c'est d'abord un mode de publication typique que l'on retrouve à l'œuvre ici. Ainsi, le texte que nous traduisons a pour titre " Heinrich Heine, das Genie der Haßliebe » et est tiré du recueil de discours, articles et recensions Über Ruhestörer. Juden in der deutschen Literatur, dans l'édition de 1993. Conformément à la pratique éditoriale de Reich-Ranicki, le recueil connaît différentes rééditions au cours desquelles il est enrichi et augmenté. Si le texte est présent dès la première édition en 1973, il porte un autre titre, "Zum Fall Heine », qu'il gardera dans la réédition de 1977. Ce n'est qu'en 1989 que le texte prend le titre et l'aspect du texte que nous traduisons. Il est réédité sous cette forme en 1993 et 2000. C'est en quelque sorte la dernière version stabilisée du texte publié, la version la plus longue et la plus riche, que nous avons choisi de traduire. Le texte, dans ses multiples versions, a donc connu différents titres. Il est paru une première fois dans l'hebdomadaire Die Zeit, le 22 septembre 1972 sous le titre « Eine Provokation und eine Zumutung », tout en étant destiné dès le début à une publication sous forme de livre. La même année, il devait en effet intégrer, sous 
le titre « Heines Genialität », l'ouvrage collectif dirigé par Wilhelm Gössmann, Geständnisse. Heine im Bewusstsein heutiger Autoren ${ }^{1}$. En 1997, à l'occasion des deux cents ans de la naissance de Heine, le texte est republié dans l'ouvrage Der Fall Heine dans sa version de 1989, mais sous le titre de 1972 « Eine Provokation und eine Zumutung ». Ce recueil rassemble trois essais représentant, selon le critique, «l'essence» de son travail sur Heine ${ }^{2}$. Si ces huit rééditions et quatre titres différents ont de quoi faire perdre son latin au philologue, d'autant que les différentes versions du texte apparaissent toutes avec la date de 1972, ils témoignent aussi de l'importance qu'accordait le critique à ce texte.

Selon Thomas Anz, les livres de Reich-Ranicki, dans leurs remaniements constants, s'exposent comme works in progress. Anz montre comment le critique envisage très souvent livre et essai de manière conjointe : l'article est très souvent pensé en fonction d'un livre à venir, dans lequel, en retour, l'unité que forme l'essai conservera malgré tout son indépendance, « la petite forme » restant pour lui « la forme de la critique $»^{3}$. Reich-Ranicki n'efface ainsi pas les redondances pouvant exister entre les trois textes qu'il décide de ne pas remanier pour son recueil de 1997 Der Fall Heine ${ }^{4}$.

Parce que Heinrich Heine est l'un des écrivains de prédilection de Reich-Ranicki, il est possible aussi d'observer à partir de la récurrence de la référence à cet auteur l'amplitude des activités du critique. En effet, Heine ne fait pas seulement l'objet d'essais dans des journaux et livres, il est également présent dans les différentes anthologies que publie Reich-Ranicki, et en particulier dans les anthologies commentatrices ou interprétatives dont Reich-Ranicki s'est fait le spécialiste. Les poèmes de Heine sont régulièrement commentés dans l'Anthologie de Francfort que le critique dirigea de 1974 à sa mort en 2013. Chaque samedi paraissent ainsi dans la Frankfurter Allgemeine Zeitung un poème et son interprétation par un critique, un universitaire ou un écrivain. Dans ce cadre, Reich-Ranicki a interprété lui-même les poèmes de Heine « Ein Jüngling liebt ein Mädchen » et « Leise zieht durch mein Gemüt » en 1997 et 19995. En 1997, il publie sur ce modèle une

1. - Indications de Marcel Reich-Ranicki, données dans Marcel Reich-Ranicki, " Nachweise und Anmerkungen », in : Id., Über Ruhestörer. Juden in der deutschen Literatur, München, dtv, 1993, p. 241.

2. - Marcel Reich-Ranicki, « Nachbemerkung », in : Id., Der Fall Heine, Stuttgart, Deutsche Verlags-Anstalt, 1997, p. 113.

3. - Thomas Anz, « Mein Beruf. Marcel Reich-Ranicki als Literaturkritiker », Literaturkritik.de Nr. 6, Juni 2010, Schwerpunkt « Literaturkritik. Zu Ehren Marcel Reich-Ranickis », https://literaturkritik.de/id/14424 (consulté le 17/11/2019).

4. - M. Reich-Ranicki, « Nachbemerkung » (1997), op. cit., p. 113-114.

5. - Cf. la bibliographie en ligne http://m-reich-ranicki.de/index.php?content=http://mreich-ranicki.de/content_biographie.html, consultée le 16 novembre 2019. 
anthologie de 44 poèmes de Heine et leurs interprétations, Ich hab im Traum geweint, en grande partie tirée de l'Anthologie de Francfort ${ }^{6}$. Enfin, c'est encore à Heine que Reich-Ranicki consacra son allocution «Heine und die Liebe » lorsqu'en février 1992 l'université d'Augsbourg lui remit le titre de docteur honoris causa, lui apportant la reconnaissance de son activité de critique littéraire, d'historien et de médiateur de la littérature par la communauté universitaire ${ }^{7}$ - reconnaissance dont on sait qu'elle lui tenait à cœur ${ }^{8}$. Heinrich Heine est donc l'écrivain qui met en évidence toutes les facettes de l'activité du critique, anthologiste, éditeur ; au croisement entre le journalisme et les lettres.

Si Heine est un personnage qui accompagne Reich-Ranicki dans tous les aspects de son activité de critique littéraire tout au long de sa carrière, c'est aussi parce qu'il lui apparaît comme un miroir de lui-même. Dans la remarque qui figure à la fin du recueil de 1997 Der Fall Heine, on peut lire « Mais personne ne m'était plus proche que Heinrich Heine. Je pouvais même de temps à autre m'identifier à lui $»^{9}$. Et plus loin :

Ma relation à Heine, mon penchant pour lui, devrais-je dire, le Juif allemand, le Juif européen, l'Européen citoyen du monde, avait plusieurs raisons et différentes causes : personnelles et générales, émotionnelles comme rationnelles - et bien sûr aussi certaines qui avaient à voir avec mon origine, mon tempérament, avec ma biographie et la mentalité qui est la mienne ${ }^{10}$.

Les derniers mots de cette remarque finale portent sur le titre initialement envisagé : «Ein Bekenntnis »11 (une confession). Ainsi parler de

6. - Cf. le registre en cours d'élaboration mis en ligne ici http://anthologie.de/ all.htm (consulté le 16/11/2019).

7. - Marcel Reich-Ranicki, « Heine und die Liebe », in : Helmut Koopmann, Henning Krauß (dir.), Heine und die Liebe / Marcel Reich-Ranicki. Streit und Humanität: Nachdenken über Lessing / Walter Jens : Vorträge und Ansprachen anlässlich der Verleihung der Ehrendoktorwürde durch die Philosophische Fakultät II der Universität Augsburg, München, Verlag Ernst Vögel, 1992, p. 23-39.

8. - Jörg Döring, “"Ja, lesen wir hier in der Gartenlaube?”. Ernesto Grassi begutachtet Marcel Reich-Ranicki für rowohlts deutsche enzyklopädie (1960) », in : Jörg Döring, Sonja Lewandowski, David Oels (dir.), rowohlts deutsche enzyklopädie: Wissenschaft im Taschenbuch, Hannover, Wehrhahn Verlag, 2017, p. 253-270.

9. - M. Reich-Ranicki, « Nachbemerkung » (1997), op. cit., p. 111. «Aber keiner stand mir näher als Heinrich Heine, ja mit ihm konnte ich mich bisweilen sogar identifizieren ».

10. — Ibid. « Meine Beziehung, ja meine Hinneigung zu Heine, dem deutschen Juden, dem jüdischen Europäer, dem europäischen Weltbürger, hatte viele Gründe und verschiedene Ursachen: persönliche und allgemeine, emotionale sowie rationale - und natürlich auch solche, die mit meiner Herkunft zu tun haben und mit meinem Temperament, mit meiner Biographie und mit meiner Mentalität. »

11. - Ibid., p. 114. 
Heine signifie pour Marcel Reich-Ranicki parler de lui-même. Le texte traduit en est un des exemples les plus flagrants.

« Heinrich Heine, das Genie der Haßliebe » est donc bien davantage que simplement représentatif de l'œuvre du critique littéraire. Il est un autoportrait. En un sens, la perpétuelle évolution du texte publié, semblant refléter les mouvements de la pensée de son auteur, Marcel Reich-Ranicki, à travers le temps et au gré des circonstances, et donc en quelque sorte « synchroniser l'œuvre et la vie »" du critique, annonçait déjà l'autoportrait. Dans sa courte postface au recueil de 1997 Der Fall Heine, Reich-Ranicki énonce deux critères au fondement de sa relation à Heine : le critère du tempérament et celui de la biographie. Ils s'appliquent sans difficulté à l'essai « Heinrich Heine, das Genie der Haßliebe ». Si en de nombreux points, notamment sur le sujet de la poésie érotique ou la pratique journalistique, l'affinité avec Heine est visible, car le texte expose des goûts communs, un certain nombre de passages mettent en place, plus radicalement, un système de lecture à double entente : ces passages se lisent à la fois comme des descriptions de l'œuvre de Heine et comme des auto-commentaires à l'effet troublant, faisant songer à l'illusion d'optique de l'image réversible du canard-lapin.

Les commentaires sur le goût de Heine pour la provocation, les jugements extrêmes et contestables, pour la tentation de la formule acérée ou bien sur sa capacité à exprimer des pensées d'une manière qui captive le plus grand nombre décrivent parfaitement le tempérament qui innerve le style de Reich-Ranicki lui-même. L'effort de compréhension de l'œuvre du poète $\mathrm{du} \mathrm{XIX}^{\mathrm{e}}$ siècle à partir des conditions historiques et psychologiques qui l'ont rendue possible apparaît dès lors aussi comme une entreprise d'auto-explication, voire d'auto-justification du critique. Certaines expressions font ainsi se télescoper les époques : "réchappé du ghetto », qui traduit « dem Getto entronnen », laisse entendre, derrière le sens historique des quartiers fermés et réservés aux Juifs ayant cours en Europe du moyen-âge au XIX ${ }^{\mathrm{e}}$ siècle, le sens qu'a pris le mot ghetto sous le nationalsocialisme : il désigne alors, comme à Varsovie où Reich-Ranicki fut expulsé13, les quartiers juifs créés par les nazis dans les territoires qu'ils occupèrent afin de confiner les populations juives avant de les envoyer dans les camps d'extermination. De même, les vocables « communistes » et « anticommunistes » de la cinquième partie du texte actualisent la question du socialisme au XIX ${ }^{\mathrm{e}}$ siècle. La question politique de l'époque et la contemporanéité de Heine et Marx sont appréhendées à travers le

12. — Voir Steffen Martus, Werkpolitik. Zur Literaturgeschichte kritischer Kommunikation vom 17. bis ins 20. Jahrhundert, Berlin, New York, De Gruyter, 2007, p. 469, qui utilise l'expression dans un sens un peu différent.

13. - Cf. l'article d'Audrey Kichelewski, « Marcel Reich-Ranicki et la Pologne : trajectoire et perceptions », dans ce numéro. 
prisme ultérieur de la guerre froide. La réflexion sur le rapport de Heine à Marx et à la révolution, déjà présente dans l'essai que Adorno consacra à Heine en 1956 - hypotexte important du texte de Reich-Ranicki - permet en même temps au critique d'articuler son propre positionnement politique, qui fait l'objet d'interrogations. En effet, Reich-Ranicki est accusé tantôt d'anticommunisme (notamment à l'Est), tantôt d'être un adepte du réalisme socialiste (à l'Ouest) ${ }^{14}$.

L'anachronisme, par ailleurs motivé par le prisme dominant de la mémoire (plutôt que de l'histoire) de la vie et de l'œuvre de Heine et justifié par la lecture rétrospective de l'œuvre de l'écrivain depuis la perspective d'Auschwitz, est ainsi l'un des ressorts principaux du dispositif de double entente instauré par le texte du critique. Il permet de superposer deux vies qu'un siècle et demi séparent mais que plusieurs éléments biographiques fondamentaux réunissent : l'origine juive, l'expérience de l'exil, le rapport ambivalent à l'Allemagne et à la langue allemande. Le motif structurant est celui de l'expérience du rejet et la perception comme parvenu, dues à la conjonction de l'origine juive et de l'expérience de l'étranger : Heine, Juif, écrivant en allemand et sur l'Allemagne depuis la France, Reich-Ranicki, Juif originaire de Pologne, écrivant sur la littérature allemande en allemand. Dans cette lecture de Heine, Reich-Ranicki se livre presque à nu, écrivant - sous couvert de l'analogie - à visage découvert, proche en cela du diagnostic qu'il formule à propos de Heine. S'il faut tenir l'analogie suggérée jusqu'au bout, alors Reich-Ranicki est lui aussi cette « figure solitaire et déchirée», dotée d'une extrême sensibilité à la douleur. Dans la réception posthume de Heine, entre rejet violent et popularité, il semble lire les réactions suscitées par ses propres textes et son activité de critique littéraire, et peut-être même anticiper l'image qui restera de lui-même après sa mort. L'« amour-haine » du titre vaut certes pour le rapport de Heine aux Allemands et aux Juifs, mais il est sans doute à lire aussi dans le sens de la réception de leur œuvre à tous deux: à la fois adulée et détestée.

Par-delà cette conscience douloureuse, l'éloge de Heine est aussi par maints aspects un éloge de Reich-Ranicki par lui-même. Les qualités et mérites attribués au poète, notamment son indépendance, le rôle déterminant qu'il joua dans l'institutionnalisation de la fonction de

14. — Lothar Müller, « Letzte Ausfahrt Klagenfurt. Wie Autoren aus der DDR die letzten Bachmann-Wettbewerbe vor dem Mauerfall gewannen », Süddeutsche Zeitung, 20/09/2019, https://www.sueddeutsche.de/kultur/klagenfurt-revisited-letzte-ausfahrtklagenfurt-1.4609083 (consulté le 17/11/2019). Cf. Maria Reinhardt, Geteilte Kritiken. DDR-Literatur und bundesrepublikanische Literaturkritik. Fallstudien zum Werk von Brigitte Reimann, Jürgen Fuchs und Hermann Kant, Heidelberg, Winter, 2018, p. 4754. Voir l'article de Stephanie Baumann, « Peter Rühmkorf, Marcel Reich-Ranicki, eine Polemik. Über Literaturkritik und mediale Öffentlichkeit in der Nachwendezeit », ainsi que notre entretien avec Ijoma Mangold dans ce numéro. 
l'écrivain, et la démocratisation de la littérature allemande qu'il initia, sont facilement transposables au domaine du critique littéraire. Ijoma Mangold souligne ainsi l'importance de Marcel Reich-Ranicki pour la reconnaissance du métier de critique littéraire. La démocratisation de la critique littéraire qui viserait à combler le fossé entre l'art et la vie apparaît comme l'un des objectifs du critique et l'une des facettes du " populaire », sous l'égide duquel nous avons souhaité placer ce cahier. Le texte sur Heine livre de manière indirecte de précieux indices sur la manière dont Reich-Ranicki concevait son métier. Ils viennent compléter les indications que le critique donna dans ses nombreux entretiens et ouvrages sur la question ${ }^{15}$. Le détour par Heine lui permet aussi implicitement de penser sa production de critique littéraire comme une œuvre : comme celle du poète, elle est inégale et composée de petites parties qui, cependant, forment un tout.

Dans un dernier temps, on pourrait s'interroger sur le type de lecture à laquelle se livre Reich-Ranicki dans cet essai sur Heine. Il semble mêler, d'un côté, la lecture savante de celui qui lit le texte avec une image de l'auteur et la conscience des procédés qu'il emploie et, de l'autre, la lecture projective de celui qui se lit lui-même dans cette image d'auteur, en une forme de lecture dite humaine. Apparentée à la lecture que Sartre propose de Flaubert dans L'Idiot de la famille, cette lecture « humaine » s'oppose à la lecture pratiquée par le marxisme, le structuralisme ou le New Criticism ${ }^{16}$. Dans ce sens, l'essai de Reich-Ranicki serait proche des fictions interauctoriales décrites par Ina Schabert comme des doubles portraits ${ }^{17}$. Dans ce type d'essai interauctorial, plutôt inattendu de la part d'un critique littéraire, se joue la constitution d'une identité auctoriale de critique au contact de celle de l'écrivain commenté. Demeure finalement la question de savoir si la lecture de soi n’y éclipse pas par endroits celle de l'auteur lu, et si l'enjeu majeur n'est pas l'affirmation du critique comme auteur.

15. - Pour n'en citer que deux : Marcel Reich-Ranicki, Über Literaturkritik, Stuttgart, München, Deutsche Verlags-Anstalt, 2002 ; Marcel Reich-Ranicki, Kritik als Beruf. Drei Gespräche, ein kritisches Intermezzo und ein Porträt, dir. Peter Laemmle, Frankfurt a. M., Fischer, 2002.

16. - Cf. Vincent Jouve, L'effet-personnage dans le roman, Paris, PUF, 1992 ; Ina Schabert, «Interauktorialität », Deutsche Vierteljahrsschrift für Literaturwissenschaft und Geistesgeschichte 57/1983, n 4, p. 680-701, p. 680-682.

17. — Ibid., p. 683. 